\title{
Mevalonic Aciduria in a Child Featuring Hepatic Fibrosis and Novel Mevalonate Kinase Mutations
}

\author{
M. Harel-Meir, Y. Bujanover", Y. Berkun, N. Goldstein and Y. Anikster \\ Department of Pediatrics B, Safra Children's Hospital, Sheba Medical Center, Sackler Faculty of Medicine, Tel Aviv \\ University, Tel Aviv, Israel
}

\begin{abstract}
Mevalonic aciduria (MVA) is an inborn error of isoprene biosynthesis caused by mevalonate kinase ( $M V K$ ) gene mutations. Described below is a case of a Palestinian MVA patient suffering from prolonged fevers as well as from hepatic fibrosis - a rare feature of MVA. Also demonstrated is a unique genotype - heterozigosity of two novel $M V K$ mutations; V8F (t25a), and F38I (t112a).
\end{abstract}

\section{INTRODUCTION}

Mevalonic aciduria (MVA) (OMIM 610377) is a rare inborn error of isoprene biosynthesis, caused by mutations in the mevalonate kinase $(M V K)$ gene. MVA features recurrent febrile episodes, developmental delay, ataxia, dysmorphism, failure to thrive, cataracts, and retinal dystrophy [1]. $M V K$ mutations are also responsible for the hyperimmunoglobulinemia D and periodic fever syndrome (HIDS) (OMIM 260920) [1], an autosomal recessive disorder characterized by recurrent febrile episodes, abdominal pain, arthritis, rash and cervical lymphadenopaty [2].

Although HIDS and MVA are considered as separate clinical entities, many patients illustrate a phenotypic spectrum, possibly related to the level of enzyme activity. Mevalonate kinase activity is less severely impaired in HIDS patients compared to its activity in MVA patients [1]. Accordingly, mevalonate is excreted in the urine of HIDS patients in low-moderate levels and only during febrile episodes, while MVA patients continuously excrete high levels of mevalonate [3]. Moreover, specific genotypes are associated with resulting different mevalonate kinase activity levels, and thus with either HIDS or MVA [4].

Most of the reported HIDS and MVA patients are of western European descent [2]. However, cases have been reported of patients from countries including Portugal [5], Italy [6] and Turkey [7]. The first Palestinian HIDS patient was recently reported [8]. The diagnosis was made through evidence of elevated IgD levels, as well as by DNA sequencing demonstrating homozigosity of the $M V K$ V377I mutation.

The following report describes a Palestinian child with hepatic fibrosis diagnosed with MVA, featuring novel mutations of the MVK gene.

*Address correspondence to this author at the Department of Pediatrics, Safra Children's Hospital, B Sheba Medical Center, Tel Hashomer, Ramat Gan 52621, Israel; Tel: 97235305006; E-mail: bujyoram@netvision.net.il

\section{CASE REPORT}

A 1.5 year old Palestinian male was referred to our hospital from the GAZA strip for the investigation of hepatosplenomegaly. He is the third of four healthy children of non-consanguineous parents (Fig. 1A). Upon detailed history, he was found to have suffered from monthly episodes of fever up to $39^{\circ} \mathrm{C}$, lasting 7-10 days, since the age of 10 weeks. In contrast to his normally developed twin sister, at the age of 1.5 years he was unable to stand, and did not use any meaningful words.

Physical examination revealed hepatomegaly, a maculopopular corporal and facial rash, and left elbow arthritis. Abdominal ultrasonography confirmed the presence of an enlarged heterogenic liver and a spleen of $9.5 \times 8.6$ $\mathrm{cm}$. Portal blood flow was normal.

Neurological examination revealed general hypotonia and a linguistic developmental delay, otherwise being a normal examination.

No pathology was found in his retina, cornea, uvea or optic discs.

Venereal disease research laboratory (VDRL) tests, as well as serology for CMV, HSV, toxoplasma, hepatitis A, B and $\mathrm{C}$ were obtained and were all found negative. Serology tests for Rubella demonstrated an immunized individual.

Levels of immunoglobulins were tested and found to be mildly elevated: IgG $1980 \mathrm{mg} / \mathrm{dL}$ (N:420-1200), IgA 189 $\mathrm{mg} / \mathrm{dL} \quad(\mathrm{N}: 12-150), \quad$ IgM $328 \quad \mathrm{mg} / \mathrm{dL} \quad(\mathrm{N}: 45-200)$. Complement levels were normal or mildly elevated. Creactive protein level was $30 \mathrm{mg} / \mathrm{dL}$. Serology for ANA, PANCA, C-ANCA, anti-LKM, anti-actin, ANF, anti-M2 and ASMA were all negative.

Liver biopsy was performed next and revealed an inflammatory lymphocytic infiltrate, associated with portal fibrosis (Fig. 2). Due to a thus suspected inflammatorymediated condition, steroid therapy was begun. This treatment diminished both arthritis and fevers, although upon weeks of follow-up had little effect on the child's general 
A

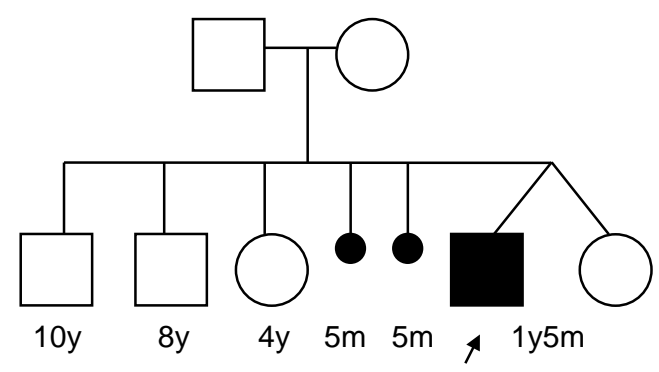

CCTAC TGG NG TCTGC TCCGGGGNAAG TCATC CT TCATGMAG ACATGC CGTGG TACAI'GG

B

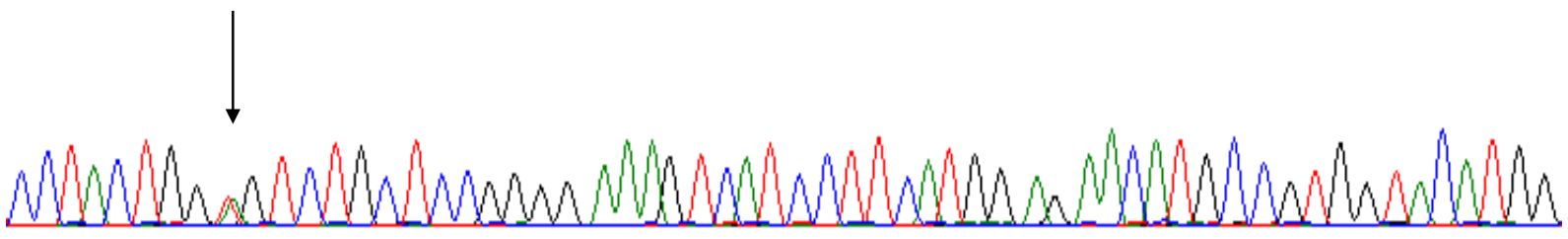

CCTAC TGG NG T CTGC TCCGGGG AAAG TCAT CCT T CATGGAG AACATG CCGTGG T

C

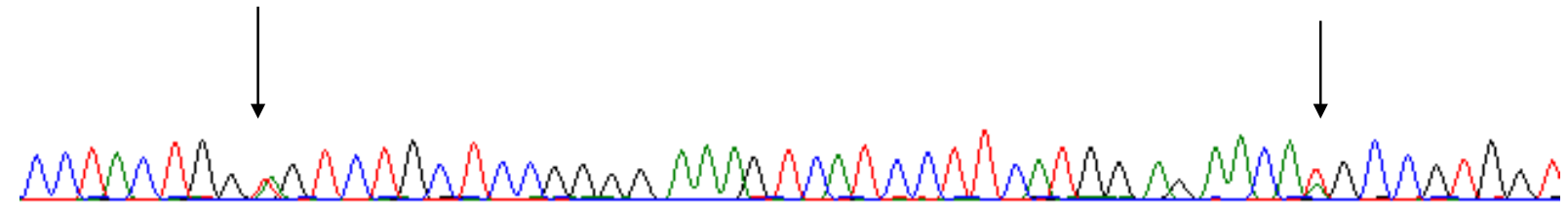

Fig. (1). (A) The patient's family pedigree. Noteworthy is the lack of consanguinity. (B) Sequencing of Exon $1 \mathrm{~F}$ of the paternal $M V K$ gene, demonstrating the V8F mutation. (C) Sequencing of Exon $1 \mathrm{~F}$ of the patient's $M V K$ gene, demonstrating compound hetrozygosity of two mutations: V8F (t25a), and F38I (t112a).

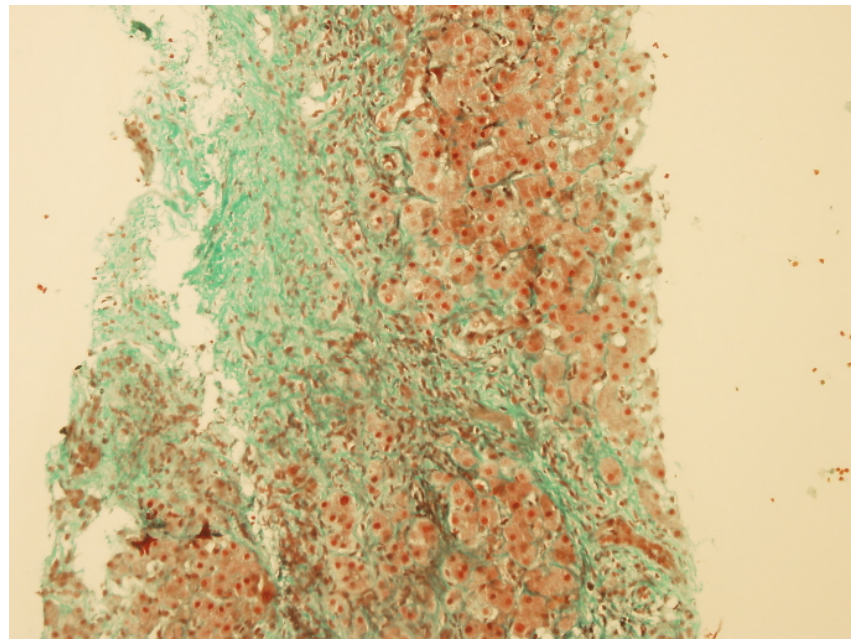

Fig. (2). A photomicrograph showing the patient's Masson trichmore stained liver tissue, demonstrating hepatic fibrosis, an unfamiliar feature of MVA.

well being. Upon the reduction of steroid dose, the fevers and arthritis reappeared. A trial of imuran treatment had no clinical effect.

Due to the combination of hepatosplenomegaly, recurrent fevers, and arthritis the diagnosis of MVA was considered and sought by urine organic-acid analysis. Mevalonate levels were $2250 \mathrm{mmol} / \mathrm{mol} \mathrm{Cr}(\mathrm{N}<0.03)$ thus confirming the diagnosis. Levels of $\operatorname{IgD}$, however, were only slightly elevated $-8.2 \mathrm{mg} / \mathrm{dL}(\mathrm{N} 0-4.5)$. Genetic sequence analysis revealed the patient to be a compound heterozygote of two novel mutations on exon 1 of the $M V K$ gene (Fig. 1C): V8F (t25a), and F38I (t112a). The patient's father was found to be the carrier of the V8F mutation (Fig. 1B). Hence the F38I mutaion is most likely maternal in origin (DNA sample for sequencing was unavailable), or less likely, a de-novo mutation.

\section{DISCUSSION}

This case demonstrates the phenotypic continuum between MVA and HIDS; this patient presented with a mild 
developmental delay, slightly elevated IgD blood levels and high urine mevalonate levels - all supporting diagnsosis of MVA [1, 2]. Still, other features of MVA such as ataxia, dysmorphic features or cataracts [1] were absent, although none are considered to be sine qua non to MVA. One might argue that ataxia has not yet developed in this patient due to his young age, however, central cataracts and unusual facies have been previously described in MVA patients as young as 2 years of age [9].

One of the diagnostic challenges presented in this patient was the presence of hepatic fibrosis in liver biopsy, which temporarily shifted the differential diagnosis to more common inflammatory hepatic diseases. Although previously described in MVA patients [10], hepatic fibrosis is far from being considered a hallmark of the disease, and is scantly described in the literature, perhaps due to the infrequent exercise of liver biopsy in these patients.

Another remarkable finding in this patient was his genotype, as he was found to be a compound heterozygote of two novel $M V K$ mutations: V8F (t25a), and F38I (t112a). Although mutations in $M V K$ have been previously described in a HIDS patient of Palestinian descent [8], our reported case is, to our knowledge, the first described Palestinian MVA patient. In general the phenotypic severity of mevalonic aciduria correlates with the activity of the enzyme mevalonate kinase [1]. In the case described, the two novel mutations cause a change from an aliphatic (V,I) amino acid to an aromatic amino acid $(\mathrm{V}, \mathrm{F})$ and vice versa in the mevalonate kinase protein structure, thus affecting its activity.

As of now, medical treatment presents the principal challenge in MVA. Previously reported treatments including statins, the tumor necrosis factor alpha (TNF- $\alpha$ ) inhibitor etanercept, and Anakinra, an interleukin-1 receptor antagonist [1] were all found to be of limited value. Recently, Neven, et al. [1] described a successful allogeneic bone marrow transplantation in an MVA patient. However, as this is a fairly new treatment, no evidence is present regarding its effect on neurological development.

In summary, this case highlights phenotypic features of MVA, such as hepatic fibrosis, and demonstrates the clinical spectrum of the disease. The reported unique genotype in this patient, as well as his unique ancestry, will hopefully enable earlier accurate diagnoses of future cases.

\section{ABBREVIATIONS}

ANA $=$ Antinuclear antibodies

Anti-LKM = Anti- liver kidney microsomal antibodies

ANF $\quad=$ Antinuclear factor
Anti-M2 = Antimitochondrial protein 2
ASMA $=$ Anti- smooth muscle antibodies
$\mathrm{C}$-ANCA = Cytoplasmic antineutrophil cytoplasmic antibodies
$\mathrm{CMV}=$ Cytomegalovirus
HIDS = Hyperimmunoglobulinemia $\mathrm{D}$ and periodic fever syndrome
$\mathrm{HSV}=$ Herpes simplex virus
IgD $=$ Immunoglobulin D
$\mathrm{IgG}=$ Immunoglobulin $\mathrm{G}$
IgM $=$ Immunoglobulin $\mathrm{M}$
IgA $=$ Immunoglobulin A
MVA $=$ Mevalonic aciduria
$M V K=$ Mevalonate kinase gene
$\mathrm{P}-\mathrm{ANCA}=$ Perinuclear antineutrophil cytoplasmic antibodies
TNF- $\alpha=$ Tumor necrosis factor alpha
VDRL $=$ Venereal disease research laboratory tests

\section{REFERENCES}

[1] Neven B, Valayannopoulos V, Quartier P, et al. Allogeneic bone marrow transplantation in mevalonic aciduria. N Engl J Med 2007; 356: 2700-3.

[2] Drenth JP, Haagsma CJ, van der Meer JW. Hyperimmunoglobulinemia $\mathrm{D}$ and periodic fever syndrome: the clinical spectrum in a series of 50 patients. Medicine (Baltimore) 1994; 73: 133-44.

[3] Houten SM, Wanders RJ, Waterham HR. Biochemical and genetic aspects of mevalonate kinase and its deficiency. Biochim Biophys Acta 2000; 1529: 19-32.

[4] Simon A, Kremer HP, Wevers RA, et al. Mevalonate kinase deficiency: evidence for a phenotypic continuum. Neurology 2004; 62: 994-7.

[5] Abreu TT. Periodic fever: the first Portuguese case-report of hyperIgD syndrome (HIDS). Acta Med Port 2004; 17: 391-4.

[6] Ostuni PA, Lazzarin P, Ongaro G, Gusi R, Todesco S, Gambari PF. Hyper-IGD syndrome: a new case treated with colchicine. Clin Rheumatol 1988; 7: 398-401.

[7] Coban E, Terzioğlu E. A patient with hyper-IgD syndrome in Antalya, Turkey. Clin Rheumatol 2004; 23: 177-8.

[8] Hammoudeh M. Hyperimmunoglobulinemia D syndrome in an Arab child. Clin Rheumatol 2005; 24: 92-4.

[9] Hoffmann G, Gibson KM, Brandt IK, Bader PI, Wappner RS, Sweetman L. Mevalonic aciduria--an inborn error of cholesterol and nonsterol isoprene biosynthesis. New Engl J Med 1986; 314: $1610-4$.

[10] Hinson DD, Rogers ZR, Hoffmann GF, et al. Hematological abnormalities and cholestatic liver disease in two patients with mevalonate kinase deficiency. Am J Med Genet 1998; 78: 408-12. 